\title{
Eubacterium rectale
}

National Cancer Institute

\section{Source}

National Cancer Institute. Eubacterium rectale. NCI Thesaurus. Code C86388.

A genus of aerobic, Gram-positive, rod shaped bacteria assigned to the phylum

Firmicutes. This species is motile, non-spore forming, esculin positive, forms butyrate,

produces abundant amounts of hydrogen, and utilizes glucose, cellobiose, melezitose,

fructose, sucrose, raffinose, melibiose, starch, and inulin, but not glycerol. E. ractale is one of the most abundant species in the human gastrointestinal tract where it ferments carbohydrates. 